\title{
MODELING THE INTERACTION BETWEEN TWO-WHEELED SELF-BALANCING VEHICLE AND ITS RIDER
}

\author{
M. CIĘŻKOWSKI \\ Białystok Technical University, Faculty of Mechanical Engineering \\ Department of Automatics and Robotics \\ ul. Wiejska 45 C, 15-351 Białystok, POLAND \\ E-mail: mciezkowski@gmail.com
}

\begin{abstract}
The paper presents a modeling method and mathematical description of a two-wheeled self-balancing vehicle and its rider. A model of the rider that was used contains a model of the ankle joint, so we could determine the interaction between the rider and the vehicle. The paper presents results of computer simulations, which show the fundamental processes during riding, such as acceleration and braking.
\end{abstract}

Key words: human-machine interaction, system control, ankle joint model, LQR, inverted pendulum.

\section{Introduction}

The rapid development of miniature electro-mechanical devices (MEMS), or ever more perfect image processing algorithms, cause the interaction between users and their devices to be no longer limited to pushing a button or pulling a lever. The modern devices are able to record, whether by camera or for example miniature accelerometers or gyroscopes, user-made gestures and interpret them as specific orders. One of such device is a two-wheeled self-balancing vehicle where acceleration is controlled by an appropriate balancing of the rider's body. A change of the rider's center of gravity causes the vehicle to lean forward, the computer records it and on that basis controls the motors. The commercial version of such a vehicle is called the Segway ${ }^{\mathrm{TM}}$ (Reference 1). The control method of such a system is very similar to the problem of stabilizing the inverted pendulum (Astrom and Murray, 2008; Ciężkowski and Siemieniako 2011). There are many papers describing the theory of TWSBV (two-wheeled self-balancing vehicle), where the main attention is focused on the problem of stability of an already leaning vehicle. In this paper, a description of both problems: the stability of the vehicle and the dislocation of the system from its equilibrium position, which is the primary goal of the TWSBV rider, will be presented.

\section{Model of the system}

The system can be divided into three basic parts: a platform with wheels, motors as the power transmission system and the rider of the vehicle. It is assumed that the measuring equipment provides all the necessary variables needed to provide control of the vehicle, working principles of the above mentioned measuring devices are not significant to the following description.

\subsection{Platform with wheels}

Figure 1 shows the physical model of the platform and wheels. The perfectly rigid disc has been taken as the model of the wheel, with mass $m_{w}$ and radius $r_{w}$. The perfectly rigid square has been taken as the model of the floor, with mass $m_{l}$ and the side length $a_{l}$. The rest of the elements of the platform, such as the battery, motor casing, etc. has been described by a cylinder with mass $m_{2}$ and radius $r_{2}$. The cylinder has been put under the floor. The mass of the steering has been neglected. 


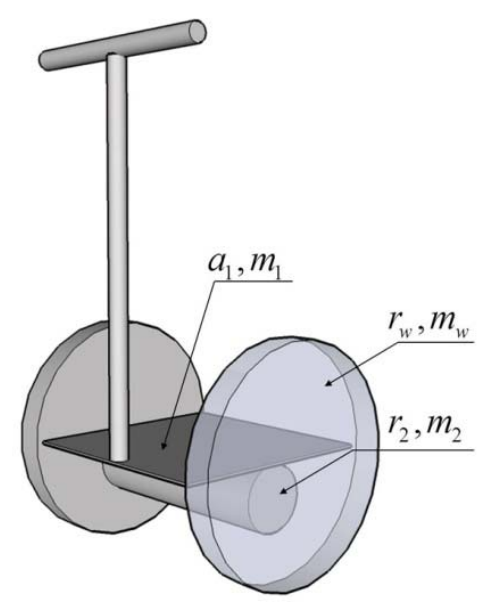

Fig.1. Physical model of the platform.

The mass of the platform $m_{p}$ is equal to $m_{1}+m_{2}$. The moment of inertia of the platform relative to the axis of the rotation passing through the center of the wheels is as follows

$$
J_{p}=\frac{1}{2} m_{2} r_{2}^{2}+m_{2} r_{2}^{2}+\frac{1}{12} m_{1} a_{1}^{2}
$$

The values of platform parameters are equal to

$$
\begin{aligned}
& m_{1}=5 \mathrm{~kg}, \quad a_{1}=0.5 \mathrm{~m}, \quad m_{2}=20 \mathrm{~kg}, \quad r_{2}=0.07 \mathrm{~m}, \quad m_{w}=5 \mathrm{~kg}, \\
& r_{w}=0.21 \mathrm{~m}, \quad m_{p}=m_{1}+m_{2} .
\end{aligned}
$$

\subsection{Motors}

The TWSBV driving system in this model is composed of two DC motors with built-in 20:1 gear whose parameters are similar to the NPC-T64 motor (Meggiolaro, 2009). Assuming zero inductance of motors (motors are relatively small) and neglecting the inductance simplify the analysis considerably. The Kirchhoff's second law for the motor can be written as follows

$$
u=I R+n k_{e} \dot{\theta}
$$

where: $u$ - voltage applied to the motor, $\dot{\theta}$ - engine (and more precisely the shaft gear) velocity, $I$ - motor current, $k_{\mathrm{e}}$ - back EMF constant, $n$ - gear ratio, $R$ - coil resistance.

Assuming that the torque generated by the engine is equal to $k_{m} I$ and the motor's viscous friction is $k_{\mathrm{w}}$, the total torque of the engine can be written

$$
M_{m}=k_{m} \frac{\left(u+k_{e}(\dot{\alpha}-n \dot{\theta})\right)}{R}-k_{w} \dot{\theta}
$$

where $\dot{\alpha}$ - the rotational speed of the platform (the motor magnets are part of the platform, therefore the rotation of the platform also generates EMF). The torque described by Eq.(2.3) has been applied in the model 
system. The turning of the vehicle is not a subject of this study. It can be assumed that the applied voltage $u$ is the same for both engines.

The motor parameters are

$$
\begin{aligned}
& n=20, \quad R=0.17 \Omega, \quad k_{m}=0.86 \frac{\mathrm{Nm}}{\mathrm{A}}, \quad k_{e}=0.044 \frac{\mathrm{Vs}}{\mathrm{rad}}, \\
& k_{w}=0.18 \frac{\mathrm{sNm}}{\mathrm{rad}}, \quad J_{m}=0.003 \mathrm{kgm}^{2}
\end{aligned}
$$

$J_{m}$ - moment of inertia of the rotor.

\subsection{Model of the rider}

The TWSBV rider controls the vehicle by leaning his body backwards and forward, so it cannot be modeled by a rigid body (for example, a rod) rigidly connected to the platform. Observing the driving technique of the Segway riders, you may notice, that they primarily bend in the ankle joint. This strategy has been also adopted in the research. The rider has been replaced by a perfectly rigid rod with mass $m$ and length $l$, connected with the platform by a joint simulating the ankle joint. This model enforces the assumption that the rider does not bend in his other joints, such as the hip. The human ankle joint (working muscles and tendons) is a very complicated and difficult system to describe by equations. It seems reasonable to assume that the model of the ankle joint can be described by two rods connected by a joint with a spring (Fig.2.). Such a model is often applied in the study of dynamics of the ankle (Loram et al., 2001; Weiss et al., 1985; Winter et al., 1998)
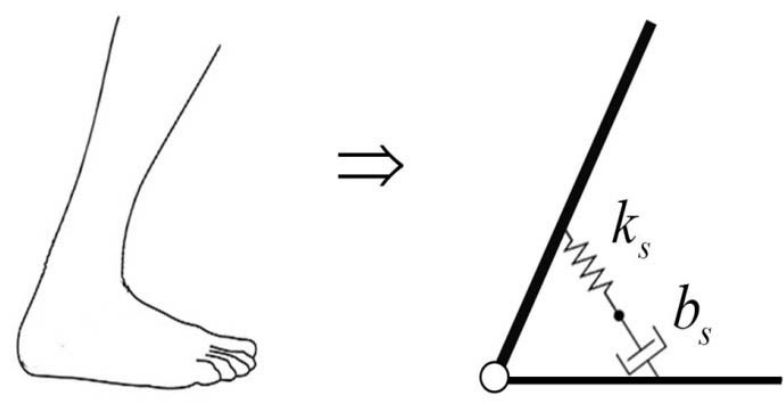

Fig.2. Physical model of the ankle joint.

According to the data contained in the papers Loram et al. (2001) and Winter et al. (1998), we can assume that for a man with mass $m=75 \mathrm{~kg}$ and height $l=1.75 \mathrm{~m}$, the elasticity coefficient $k_{\mathrm{s}}$ and damping coefficient $b_{s}$ are

$$
k_{s}=1570 \frac{\mathrm{Nm}}{\mathrm{rad}}, \quad b_{\mathrm{s}}=350 \frac{\mathrm{Nms}}{\mathrm{rad}} .
$$

It should be noted that these coefficients are already set for two ankles instead of one. It is also assumed that the rider will not detach his feet from the platform. If the rider exerts the torque $T_{u}$ on the ankle joint and deflection of the joint is $\phi$, the total torque generated by the ankle joint amounts to

$$
M_{s}=T_{u}-k_{s} \phi-b_{s} \dot{\phi}
$$




\subsection{Model of the vehicle with the rider}

Using the model of the platform, engines and the rider, the mathematical description of the system can be formulated. Figure 3 shows the physical model of the system. The model is placed in a gravitational field with a value of acceleration $g$. The system has been described by the Lagrange formalism.
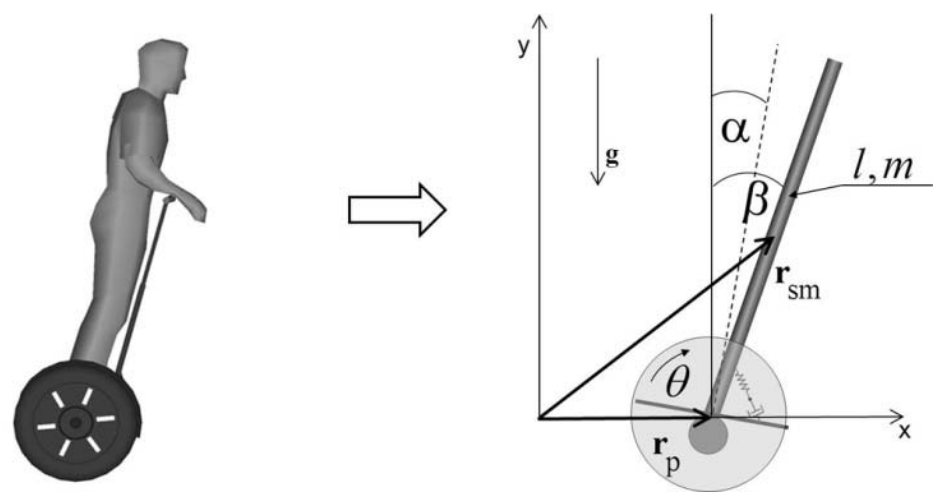

Fig.3. Physical model of the system

The position of the platform is described by a vector

$$
r_{p}=\left(\theta r_{w}, 0\right)
$$

where: $\theta$ - wheel rotation angle.

Describing the position of the platform by the angle $\theta$ follows from the assumption that the motion takes place without slipping.

The position of the rider's mass center

$$
r_{s m}=r_{p}+\frac{1}{2} l(\sin (\beta), \cos (\beta))=\left(\theta r_{w}+\frac{1}{2} l \sin (\beta), \frac{1}{2} l \cos (\beta)\right)
$$

where: $\beta$-the rider's inclination angle with respect to the $y$-axis.

The kinetic energy of the rider is the sum of the translational kinetic energy of the mass center and the rotational kinetic energy relative to the mass center

$$
T_{1}=\frac{1}{2} m \dot{\mathrm{r}}_{s m}^{2}+\frac{1}{2} \frac{1}{12} m l^{2} \dot{\beta}^{2}=\frac{1}{6} \dot{\beta}^{2} l^{2} m+\frac{1}{2} \dot{\beta} \dot{\theta} l m \cos (\beta) r_{w}+\frac{1}{2} \dot{\theta}^{2} m r_{w}^{2}
$$

The kinetic energy of the platform

$$
T_{2}=\frac{1}{2} J_{p} \dot{\alpha}^{2}+\frac{1}{2} \dot{\theta}^{2} m_{p} r_{w}^{2}
$$

The kinetic energy of the wheels and the motor's rotors

$$
T_{3}=\dot{\theta}^{2} n^{2} J_{s}+\frac{3}{2} \dot{\theta}^{2} m_{w} r_{w}^{2}
$$


Potential energy of the rider, the platform and the spring in the ankle joint

$$
V=\frac{1}{2} g l m \cos (\beta)-g r_{2} m_{2} \cos (\alpha)+\frac{1}{2} k_{s}(\beta-\alpha)^{2} .
$$

The Lagrangian takes the form

$$
L=T_{1}+T_{2}+T_{3}-V .
$$

Using Eqs (2.11), (2.3) and (2.4), three Euler-Lagrange equations take the form

$$
\begin{aligned}
& \frac{d}{d t} \frac{\partial L}{\partial \dot{\theta}}-\frac{\partial L}{\partial \theta}=2 M_{m}, \\
& \frac{d}{d t} \frac{\partial L}{\partial \dot{\alpha}}-\frac{\partial L}{\partial \alpha}=-2 M_{m}+b_{s}(\dot{\beta}-\dot{\alpha})-T_{u}, \\
& \frac{d}{d t} \frac{\partial L}{\partial \dot{\beta}}-\frac{\partial L}{\partial \beta}=-b_{s}(\dot{\beta}-\dot{\alpha})+T_{u} .
\end{aligned}
$$

The obtained equations are the equations of motion of the system.

\section{Calculation of motor control}

The control system of the vehicle is provided by applying an appropriate voltage $u$ to the motors. The electronic system IMU, placed on the vehicle, measures the angle and speed of inclination of the platform $(\alpha$ and $\dot{\alpha})$, and another sensor such as the encoder measures the velocity $\dot{\theta}$. The voltage $u$ will thus depend only on these parameters. Calculation of the voltage $u$ requires the assumption that the rider is not in a bent position, i.e.: $\beta=\alpha$. The angle $\beta$ must be equal to $\alpha$, otherwise the system will not be stable. According to the assumptions that is: $\beta=\alpha, \dot{\beta}=\dot{\alpha}, T_{u}=0$, Eq.(2.13) takes the form

$$
\frac{d}{d t} \frac{\partial L}{\partial \dot{\alpha}}-\frac{\partial L}{\partial \alpha}=-2 M_{m} \quad \text { for } \quad \beta=\alpha, \quad \dot{\beta}=\dot{\alpha}, \quad T_{u}=0
$$

Equation (3.1) is a function of the following variables $\alpha, \dot{\alpha}, \dot{\theta}$. Velocity $\dot{\theta}$ occurs only in the expression $M_{m}$ and describes the friction of the motor. If the friction of the motor is neglected, then Eq.(3.1) will depend only on $\alpha, \dot{\alpha}$. The equation will be used to determine voltage control using LQR controller. In the next step, the neglected friction will be compensated through an amendment to the voltage $u$.

\section{1. $L Q R$ controller}

To determine the motor control system, a linear-quadratic regulator was used. Using Eqs (2.3) and (3.1) you can write the state equation, which takes the form

$$
\dot{x}=A x+B u
$$

where: $x=(\alpha, \dot{\alpha})^{T}, A$ and $B$ - state matrix and input matrix, $u$ - motor voltage.

Defining a quadratic cost function as 


$$
J=\frac{1}{2} \int_{0}^{\infty}\left(Q_{11} \alpha^{2}+Q_{22} \dot{\alpha}^{2}+R u^{2}\right) d t
$$

and taking according to Bryson's rule (Bryson et al., 1969)

$$
Q_{11}=\frac{1}{0.2^{2}}, \quad Q_{22}=\frac{1}{2^{2}}, \quad R=1
$$

the voltage stabilizing the TWSBV can be calculated

$$
u_{s t a b}=-k_{1} \alpha-k_{2} \dot{\alpha}=44.35 \alpha+12.54 \dot{\alpha} \text {. }
$$

It is expected that the system does not change its velocity when the rider is not in a bent position. The friction of the motor has to be compensated (previously mentioned amendment). The unchanged velocity condition, according to Newton's first law (from Eq. (2.3)): $M_{m}=k_{m} \frac{\left(u-k_{e} n \dot{\theta}\right)}{R}-k_{w} \dot{\theta}=0$.

With this condition, you can calculate $u$

$$
u_{\dot{\theta}}=\left(n k_{e}+\frac{R k_{w}}{k_{m}}\right) \dot{\theta}=0.91558 \dot{\theta}
$$

Taking into account the result of Eqs (3.4)-(3.5), the final form of the control voltage is

$$
u=u_{\text {stab }}+u_{\dot{\theta}}=44.35 \alpha+12.54 \dot{\alpha}+0.91558 \dot{\theta} .
$$

\subsection{Velocity control}

To solve Eqs (2.12)-(2.14) numerically, we still need to find a torque $T_{u}$ acting on the ankle joint to achieve the desired velocity. The simplest way to achieve this is to use the dependence of the torque $T_{u}$ on the difference between the reference velocity and the actual velocity of the vehicle. It can be assumed

$$
T_{u}=C_{l}\left(\dot{\theta}_{S E T}-\dot{\theta}\right)
$$

where: $C_{1}$ - controller gain,

$\dot{\theta}_{S E T}$ - reference velocity

Using the formula-Eq.(3.7), the Euler-Lagrange equations from two Eqs (2.12)-(2.14), can be solved numerically. This system takes the form

$$
\begin{aligned}
& \ddot{\alpha}=-\frac{1}{J_{p}}\left\{\dot{\alpha} b_{s}-\dot{\beta} b_{s}+C_{1}\left(\dot{\theta}_{S E T}-\dot{\theta}\right)+g m_{2} r_{2} \sin (\alpha)+\alpha k_{s}-\beta k_{s}+\right. \\
& \left.+\left(2 \dot{\theta}\left(k_{m}\left(k_{3}-n k_{e}\right)-R k_{w}\right)-2 k_{m}\left(\dot{\alpha}\left(k_{2}-k_{e}\right)+\alpha k_{1}\right)\right) / R\right\},
\end{aligned}
$$




$$
\begin{aligned}
& \ddot{\theta}=\left\{\frac { 1 } { 2 } R r _ { w } \left(12 \cos (\beta)\left((\dot{\beta}-\dot{\alpha}) b_{s}+C_{l}\left(\dot{\theta}-\dot{\theta}_{S E T}\right)+(\beta-\alpha) k_{s}\right)+\right.\right. \\
& \left.-3 g l m \sin (2 \beta)+4 \dot{\beta}^{2} l^{2} m \sin (\beta)\right)-8 l k_{m}\left(\dot{\alpha}\left(k_{2}-k_{e}\right)+\alpha k_{l}\right)+ \\
& \left.+8 \dot{\theta} l\left(k_{m}\left(k_{3}-n k_{e}\right)-R k_{w}\right)\right\} / l\left\{R\left(8 n^{2} J_{m}+r_{w}^{2}\left(-3 m \cos ^{2}(\beta)+4 m_{p}+12 m_{w}+4 m\right)\right)\right\}, \\
& \ddot{\beta}=\frac{3}{2 l^{2}}\left\{\frac{\cos (\beta) r_{w} l 6 l\left(k_{m}\left(\dot{\alpha}\left(k_{2}-k_{e}\right)+\alpha k_{l}\right)+\dot{\theta}\left(k_{m}\left(n k_{e}-k_{3}\right)+R k_{w}\right)\right)}{16 n^{2} R J_{m}+R r_{w}^{2}\left(-3 m \cos (2 \beta)+8 m_{p}+24 m_{w}+5 m\right)}+\right. \\
& +\frac{\cos (\beta) r_{w} R r_{w} 12 \cos (\beta)\left((\dot{\alpha}-\dot{\beta}) b_{s}+C_{l}\left(\dot{\theta}_{S E T}-\dot{\theta}\right)+(\alpha-\beta) k_{s}\right)}{16 n^{2} R J_{m}+R r_{w}^{2}\left(-3 m \cos (2 \beta)+8 m_{p}+24 m_{w}+5 m\right)}+ \\
& +\frac{\cos (\beta) r_{w} R r_{w}\left(3 g l m \sin (2 \beta)-4 \dot{\beta}^{2} l^{2} m \sin (\beta)\right)}{16 n^{2} R J_{m}+R r_{w}^{2}\left(-3 m \cos (2 \beta)+8 m_{p}+24 m_{w}+5 m\right)}+ \\
& \left.+\frac{2(\dot{\alpha}-\dot{\beta}) b_{s}+2 C_{l}\left(\dot{\theta}_{S E T}-\dot{\theta}\right)+g l m \sin (\beta)+2(\alpha-\beta) k_{s}}{m}\right\} .
\end{aligned}
$$

The equations obtained are the equations of motion of the system.

\section{Numerical simulation results}

The last thing to do is to determine the parameter $C_{1}$ in Eq.(3.7).

\subsection{The choice of setting $\mathrm{C} 1$}

The condition to get the reference velocity of the vehicle is: $C_{1}>0$. Obviously, it is important to accelerate the vehicle in a reasonable time and not to overshoot the system, since oscillations of the state vector will be manifested. Figures 4,5 and 6 show the graphs of linear velocity of the TWSBV $\left(V=3.6 \dot{\theta} r_{w}[\mathrm{~km} / \mathrm{h}]\right)$ as a function of time for various $C_{1}$ and various $V_{S E T}=3.6 \dot{\theta}_{S E T} r_{w}[\mathrm{~km} / \mathrm{h}]$.

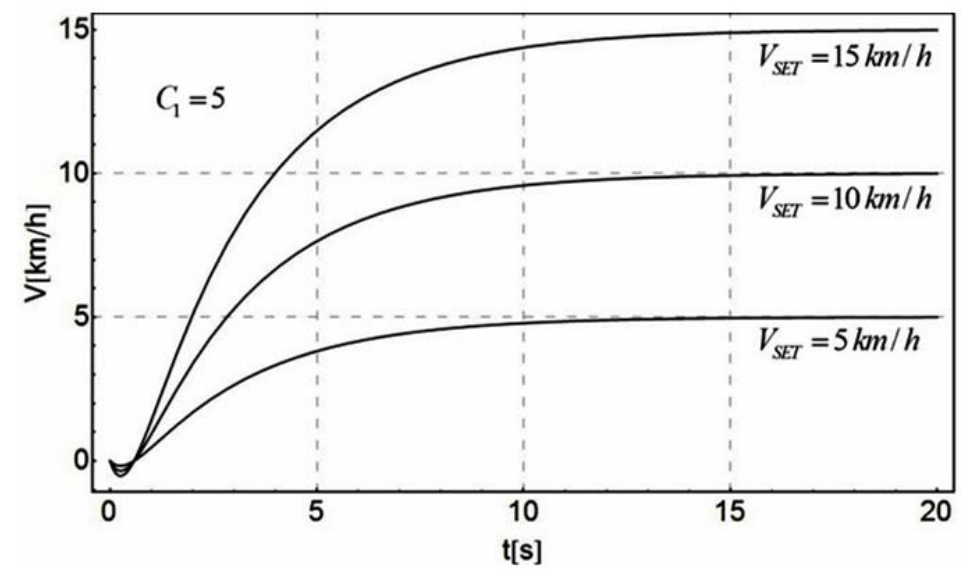

Fig.4. Velocity of a vehicle for $C 1=5 \mathrm{mNrad} / \mathrm{s}$. 


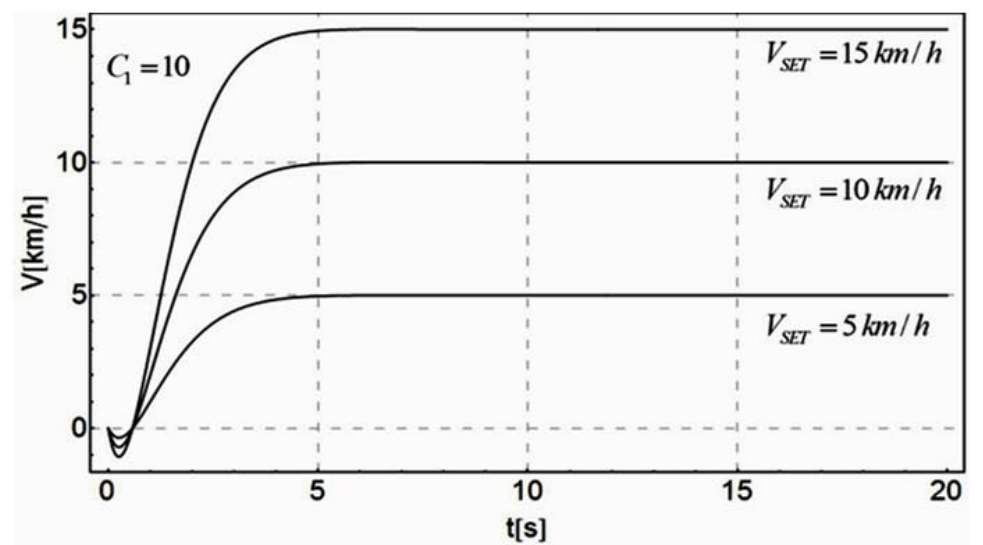

Fig.5. Velocity of a vehicle for $\mathrm{Cl}=10 \mathrm{mNrad} / \mathrm{s}$.

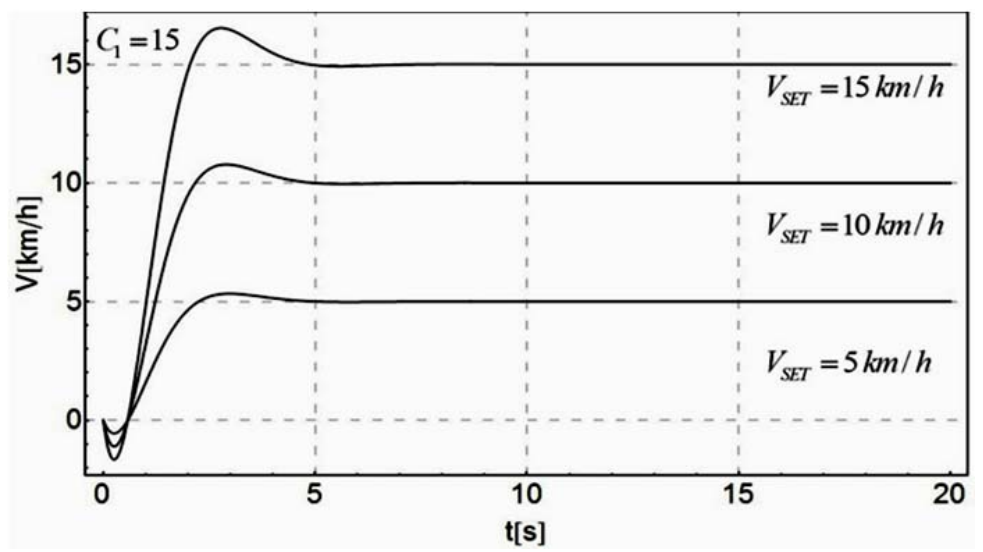

Fig.6. Velocity of a vehicle for $C 1=15 \mathrm{mNrad} / \mathrm{s}$.

By analyzing Figs 4-6 it can be concluded that the weak point of Eq.(3.7) is the constant value of the parameter $C_{1}$. For a fixed $C_{1}$, the system indeed reaches a certain velocity, but it takes always the same time - it does not matter if it reaches a top speed of 5 or $15 \mathrm{~km} / \mathrm{h}$. It would be good to be able to control the acceleration time, and this problem is the subject of further research. It is reasonable to assume that the time needed to accelerate oscillates around $5 \mathrm{~s}$ and therefore, for further simulations $C_{1}=10 \mathrm{mNrad} / \mathrm{s}$ will be taken.

\subsection{Acceleration to the reference velocity}

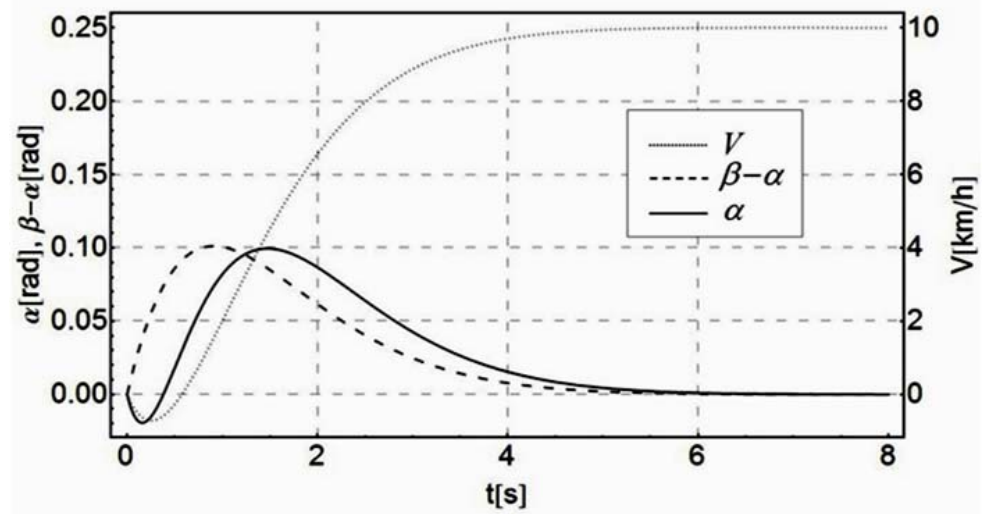

Fig.7. The inclination of the vehicle and the rider during acceleration. 
Figure 7 shows a graph of inclination of the platform $\alpha$, inclination of the rider relative to the platform $(\beta-\alpha)$ and vehicle velocity $V$ as a function of time during acceleration to $V_{S E T}=10 \mathrm{~km} / \mathrm{h}$. As shown in Fig.7., the reference velocity was reached after about $5 \mathrm{~s}$. At the beginning of the process, an interesting behavior of the system can be observed. It may be noted that the rider leans forward, and at this time the platform leans back and the vehicle begins to ride backwards. This can be easily explained by the principle of conservation of angular momentum: angular momentum at the time $t=0$ is zero and when the rider leans forward, the platform has to lean backward because the angular momentum must still be zero. The measurement system registered the minus angle of the platform and the driver applied voltage to the motor in order to straighten the platform- the platform is going back for a while. As can be seen later, the platform leans forward ("spring" in the ankle joint rotates the platform) and then the rider and the platform are upright, and the vehicle moves with a fixed velocity $V_{S E T}$.

\subsection{Braking}

Figure 8 shows the simulation results of the $\alpha,(\beta-\alpha)$ and the vehicle velocity $V$ as a function of time during braking from $V=10 \mathrm{~km} / \mathrm{h}$ to $V_{S E T}=0$.

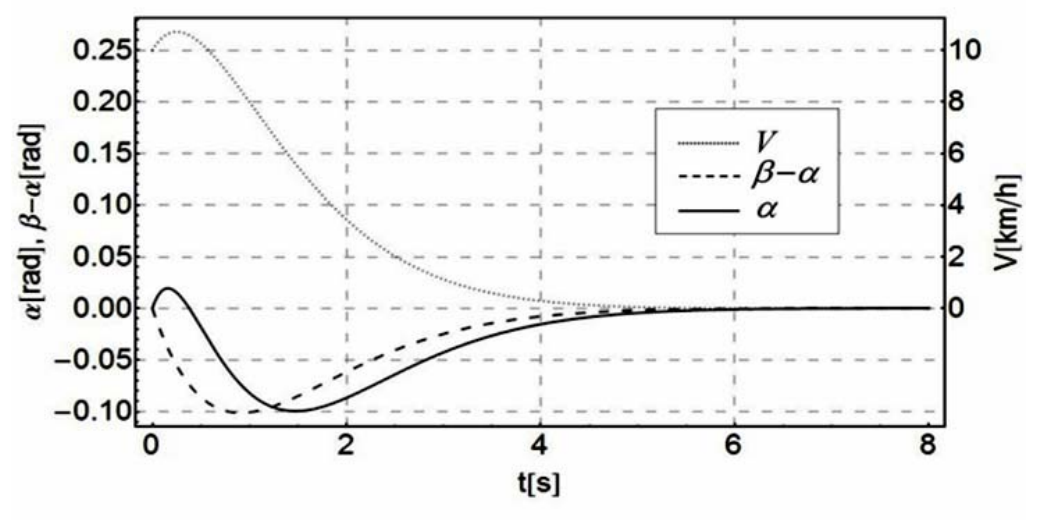

Fig.8. The inclination of the vehicle and the rider during braking.

As can be seen there are analogical processes during acceleration.

\subsection{Simulation in the presence of random noise}

The simulation results presented above assume that the measurement of parameters that determine the voltage $u$ (that is: $\alpha, \dot{\alpha}, \dot{\theta}$ ) is perfect. In the real world, random disorders cannot be eliminated. To make the system more realistic, numerical simulation in the presence of random disturbances was performed. The voltage $u$ then takes the form

$$
u=44.35\left(\alpha+\varepsilon_{1}\right)+12.54\left(\dot{\alpha}+\varepsilon_{1}\right)+0.91558\left(\dot{\theta}+\varepsilon_{2}\right)
$$

Variables $\varepsilon_{1}, \varepsilon_{2}$ stand for random disturbances and they are assumed to follow normal distribution with the density function respectively 


$$
\phi\left(\varepsilon_{1}\right)=\frac{1}{\sigma_{1} \sqrt{2 \pi}} e^{\frac{-\varepsilon_{1}{ }^{2}}{2 \sigma_{1}^{2}}}, \quad \phi\left(\varepsilon_{2}\right)=\frac{1}{\sigma_{2} \sqrt{2 \pi}} e^{\frac{-\varepsilon_{2}^{2}}{2 \sigma_{2}^{2}}} .
$$

The average values of $\varepsilon_{1}, \varepsilon_{2}$ are zero. The perturbation changes randomly at each simulation time step. The standard deviation was adopted as follows: $\sigma_{1}=0.05, \sigma_{2}=0.5$. In the case of the angle $\alpha$, noise with standard deviation $\sigma_{l}=0.05$ (about $2.8^{\circ}$ ) can be considered as significant. The noise of velocity was adopted even greater: $\sigma_{2}=0.5$ (about $28^{\circ} / \mathrm{s}$ ). The results of these simulations are illustrated in Fig.9.

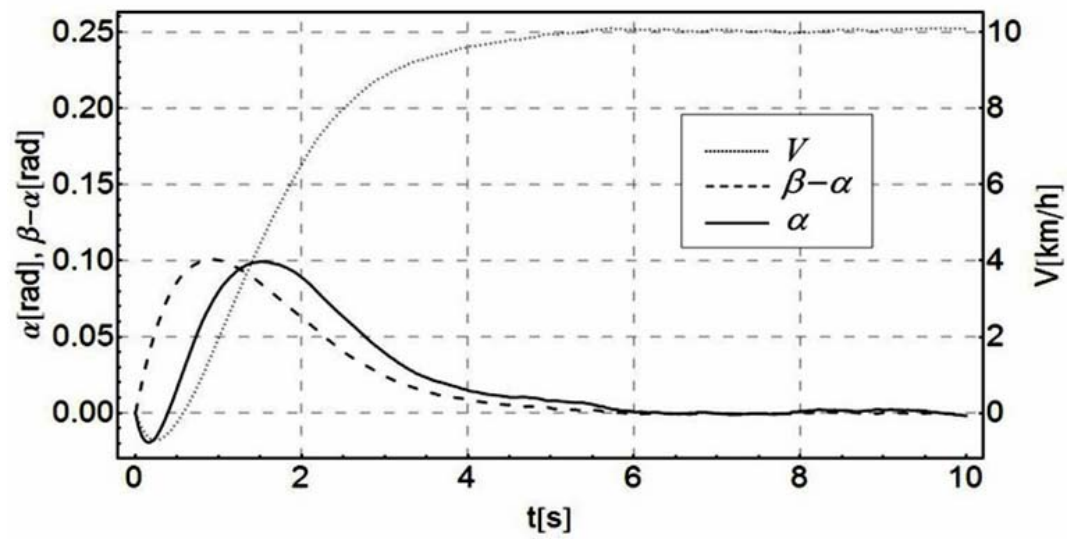

Fig.9. The vehicle and the rider inclination during acceleration.

As you can see in Fig.9. the system is measurement error-proof in the selected range.

\section{Summary}

The main objective of this study was to show that the rider-vehicle interaction can be simulated using a simple human model. This goal has been achieved and the ankle model provides promising results. This paper, however, does not answer the question: whether this model describes the reality properly. To answer that an experiment with the TWSBV should be conducted and the results should be compared with a simulation. It may turn out that the controller described by Eq.(3.7) is not real, that means that a human behaves different "by nature" and then using the experiment data the correct control system will be determined. It may turn out that using the described model, the operation of the TWSBV can be optimized. An explanation of the above-mentioned problems is the current topic of the author's research.

\section{Nomenclature}

$a_{1}-$ side length of the floor

$b_{\mathrm{s}}-$ damping coefficient of ankle joint

$I$ - motor current

$J_{m}-$ moment of inertia of the rotor

$J_{p}-$ moment of inertia of the platform

$k_{e}$ - back EMF constant

$k_{m}-$ motor torque constant

$k_{\mathrm{s}}$ - elasticity coefficient of ankle joint

$k_{w}$ - motor viscous constant

$l$ - height of the rider 


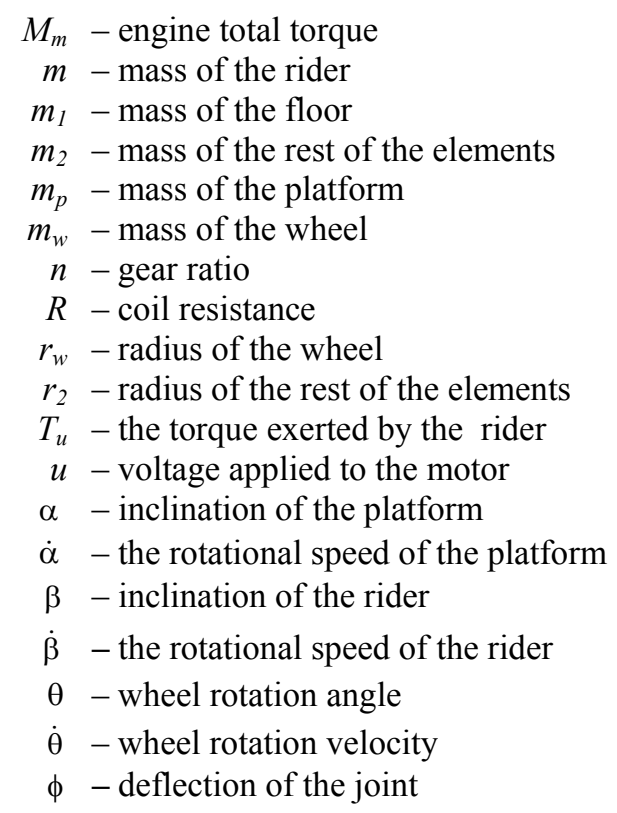

\section{References}

Astrom K.J. and Murray R.M. (2008): Feedback Systems: An Introduction for Scientists and Engineers. - Princeton: University Press.

Bryson A.E. and Ho Y.C. (1969): Applied Optimal Control. - Blaisdell, Waltham, MA.

Ciężkowski M. and Siemieniako F. (2012): Closed-loop position control of inverted pendulum. - Scientific and Didactic Equipment, Vol. XVII Nr 1/2012, pp.57-62.

Loram I.D., Kelly S.M. and Lakie M. (2001): Human balancing of an inverted pendulum: is sway size controlled by ankle impedance? - Journal of Physiology, 532.3, pp.879-891.

Meggiolaro M.A. (2009): RioBotz Combat Robot Tutorial. - CreateSpace.

Reference 1, http://www.segway.com

Weiss P.L. (1985): Position Dependence of Ankle Joint Dynamics. - McGill University.

Winter D.A., Patla A.E., Prince F., Ishac M. and Gielo-Perczak K. (1998): Stiffness Control of Balance in Quiet Standing. - Journal of Neurophysiology 80:1211-1221. 